\title{
THE SYMMETRIC DERIVATION BASIS MEASURE AND THE PACKING MEASURE
}

\author{
SANDRA MEINERSHAGEN
}

(Communicated by R. Daniel Mauldin)

\begin{abstract}
The packing measure as defined by S. J. Taylor for continuous, monotone functions $h$ and the measure generated by the symmetric derivation basis measure using $h$ are shown here to be the same for subsets of the real line.
\end{abstract}

Brian S. Thomson suggested comparing the measure generated by the symmetric derivation basis in [1] with the packing measure defined by $\mathrm{S}$. James Taylor. It is shown in this paper that on the line they are identical.

The measure generated by the symmetric derivation basis is defined as follows:

DEFINITION 1. Let $h$ be a monotone increasing function and continuous such that the range of $h$ is in $[0, \infty)$. Then for a positive real function $\delta(\cdot)$ on the real line, let

$$
\beta_{\delta}^{s}=\{(x, I): x \text { is the midpoint of } I \text { and } I \subset(x-\delta(x), x+\delta(x))\}
$$

and let

$$
D^{s}=\left\{\beta_{\delta}^{s}: \delta(\cdot) \text { is a positive function on } R\right\}
$$

Then

$$
\begin{aligned}
& V\left(h \circ m, \beta_{\delta}^{s}[E]\right)=\sup \left\{\sum_{\pi[E]} h\left(\left|I_{i}\right|\right): \pi\right. \text { is a collection of } \\
& \text { nonoverlapping intervals in } \beta_{\delta}^{s} \text { and, } \\
& \qquad \text { if }(x, I) \in \pi[E], \text { then } x \in E\}
\end{aligned}
$$

and

$$
V\left(h \circ m, D^{s}[E]\right)=\inf _{\delta} V\left(h \circ m, \beta_{\delta}^{s}[E]\right) .
$$

When $V\left(h \circ m, D^{s}[E]\right)$ is being considered as a measure rather than a variation, we write

$$
(h \circ m)_{s}(E)=V\left(h \circ m, D^{s}[E]\right) .
$$

The packing measure is defined as follows.

Received by the editors June 3, 1987.

1980 Mathematics Subject Classification (1985 Revision). Primary 28A10.

Key words and phrases. Derivation basis, packing measure. 
DEFINITION 2. Let $h$ be defined as above and let $B(x, r)=(x-r, x+r)$. Then

$$
(h-P)(E)=\lim _{\delta \downarrow 0} \sup \left\{\sum_{i} h\left(2 r_{i}\right): B\left(x_{i}, r_{i}\right) \text { are disjoint, } x_{i} \in E, r_{i}<\delta\right\}
$$

and

$$
(h-p)(E)=\inf \left\{\sum(h-P)\left(E_{i}\right): E \subset \bigcup_{i}\left(E_{i}\right)\right\}
$$

Note first that since $(h-p)(E)=\inf \left\{\lim (h-P)\left(E_{n}\right): E_{n} \uparrow E\right\}$, we can approximate $(h-p)$ with $(h-P)[2]$.

The following observation is almost obvious.

OBSERVATION. $(h \circ m)_{s}(E) \leq(h-p)(E)$.

PROOF. It suffices to show that $(h \circ m)_{s}(E) \leq(h-P)(E)$. For a given $\delta(\cdot)$ and $\pi \subset \beta_{\delta}^{s}, \sum_{\pi[E]} h(|I|)$ is a finite sum. Therefore, if $\delta(\cdot)$ is defined to be a constant $\delta$,

$$
V\left(h \circ m, \beta_{\delta}^{s}[E]\right) \leq \sup \left\{\sum_{i=1}^{\infty} h\left(2 r_{i}\right): B\left(x_{i}, r_{i}\right) \text { are disjoint, } x_{i} \in E, r_{i}<\delta\right\} \text {. }
$$

Since $(h \circ m)_{s}(E)$ is the infimum over all positive functions $\delta(\cdot)$,

$$
(h \circ m)_{s}(E) \leq(h-P)(E) \text {. }
$$

It follows that if $(h-p)(E)=0$, then $(h \circ m)_{s}(E)=0$. Therefore we shall consider when the packing measure is positive.

THEOREM. For every set $E \subset R,(h \circ m)_{s}(E)=(h-p)(E)$.

ProOF. Fix $E \subset R$. Let $M>0$ be given with $M<(h-p)(E)$. Let $\delta(\cdot)$ be any positive function, and $E_{n}=\{x \in E: \delta(x)>1 / n\}$ for $n=1,2, \ldots$ Then $E_{n} \uparrow E$. By Lemma 5.1 part (v) in [2], $(h-p)\left(E_{n}\right) \rightarrow(h-p)(E)$. Therefore, there exists an $N$ such that $(h-p)\left(E_{N}\right)>M$. Therefore $(h-P)\left(E_{N}\right)>M$. Let $\delta<1 / N$. Then, there exists $\left\{B\left(x_{i}, r_{i}\right)\right\}_{i=1}^{\infty}$ where $x_{i} \in E_{N}, r_{i}<\delta$ and $\sum_{i=1}^{\infty} h\left(2 r_{i}\right)>M$. Since $r_{i}<\delta<1 / N<\delta(x)$ for all $x \in E_{N}$,

$$
\sum_{i=1}^{\infty} h\left(2 r_{i}\right) \leq V\left(h \circ m, \beta_{\delta}^{s}\left[E_{N}\right]\right)
$$

since $V\left(h \circ m, \beta_{\delta}^{s}\left[E_{N}\right]\right)$ is a supremum over all finite sums. Therefore $M<$ $V\left(h \circ m, \beta_{\delta}^{s}\left[E_{N}\right]\right) \leq V\left(h \circ m, \beta_{\delta}^{s}[E]\right)$. Since $\delta(\cdot)$ was arbitrary, $M<(h \circ m)_{s}(E)$. Since $M$ was an arbitrary number less than $(h-p)(E)$ and by the observation that $(h \circ m)_{s}(E) \leq(h-p)(E)$, it follows that $(h \circ m)_{s}(E)=(h-p)(E)$.

\section{REFERENCES}

1. S. Meinershagen, Derivation bases and the Hausdorff measure, Real Analysis Exchange (sur mitted).

2. S. J. Taylor and C. Tricot, Packing measure, and its evaluation for a Brownian path, Trans. Amer. Math. Soc. 288 (1985), 679-699.

Department of Mathematics, NORThWEST Missouri State UNiVersity, MaRYVILLE, MISSOURI 64468 